\title{
Discussion of Intelligent Manufacturing of Complicated Electronic Equipment in the Informatization Age---Innovation and Challenges
}

\author{
Wenge XING ${ }^{1 \mathrm{a}}$, Kecun $\mathrm{BEN}^{1 *}$ \\ ${ }^{1}$ Nanjing Research Institute of Electronics Technology, Nanjing, China
}

\begin{abstract}
In the background of global manufacturing industry moving towards digitization, networking and intelligence, based on the deep integration of new generation information communication technology and advanced manufacturing technology, the manufacturing characteristics of complex electronic equipment such as variety, changing batch, updating and replacing quickly, multi-disciplinary coupling and supply chain complex are analyzed. On this basis, the innovation characteristics of complex electronic equipment in the whole life cycle of product manufacturing such as technology application, R\&D mode, production organization and management decision are studied. The main challenges in the rapid development of intelligent manufacturing of complex electronic equipment are further pointed out. In the future, with the continuous progress of information technology and advanced manufacturing technology, the current challenges of intelligent manufacturing of complex electronic equipment will be solved.
\end{abstract}

\section{Introduction}

At present, human society has entered the information age. The integration and innovation of new generation information technology and advanced manufacturing technology create conditions for the rise of intelligent manufacturing. The core purpose of intelligent manufacturing is to help enterprises improve their core competitiveness and profitability by reducing costs and increasing efficiency, saving energy and reducing consumption, improving product quality, enhancing product added value, shortening product marketing cycle, meeting customers' personalized needs, and seeking benefits from services. Complex electronic equipment is widely used in communication, detection, navigation, countermeasure and other electronic systems, and it is the carrier of information detection, storage, processing and transmission. In the background of information age, the development of intelligent manufacturing of complex electronic equipment has both innovation and challenge. Based on the analysis of the characteristics of complex electronic equipment manufacturing, this paper studies the innovative characteristics of complex electronic equipment intelligent manufacturing, discusses the current challenges of complex electronic equipment intelligent manufacturing, and looks forward to the future development direction.

\section{Characteristics of manufacturing complicated electronic equipment}

Complicated electronic equipment involves a number of disciplines and integrates parts and subsystems of mechanical, control, electronic, hydraulic, pneumatic, and software disciplines etc. Complicated electronic equipment's scientific research and production is characterized by complicated process management, varying technical statuses, multiple types, small batches, and cross discipline fusion etc. Therefore, higher requirements apply in aspects of product manufacturing full life cycle status control and management, flexible manufacturing, delivery efficiency, multi-discipline design optimization, and system integration capability etc. These are mainly reflected in the following 12 :

- Integration of scientific research, production, and guarantee requires full life cycle control and management capability from $R \& D$ to delivery of products.

- Multiple types, multiple batches, small batch quantities, and order type production requires more flexible intelligent production organization mode.

- Complicated electronic equipment has quick pace of renewal and requires quicker and more accurate response by the manufacturing platform.

- It involves multi-disciplinary, and needs to have a higher ability of multi-disciplinary comprehensive design optimization. 
- The information of process control and manufacturing management is complex and diverse, so it needs stronger cross system and cross platform integration capability.

\section{Innovative features of intelligent manufacturing of complicated electronic equipment}

Intelligent manufacturing of complex electronic equipment has many innovative features in the application of new technology, research and development mode, production organization and so on. In depth understanding of these innovative features plays an important role in grasping the key to the construction of intelligent manufacturing platform and system for complex electronic equipment.

\subsection{Innovation by application of new technologies}

A series of key technologies are needed to support the intelligent manufacturing of complex electronic equipment. The most important support is the integration and innovative application of advanced manufacturing

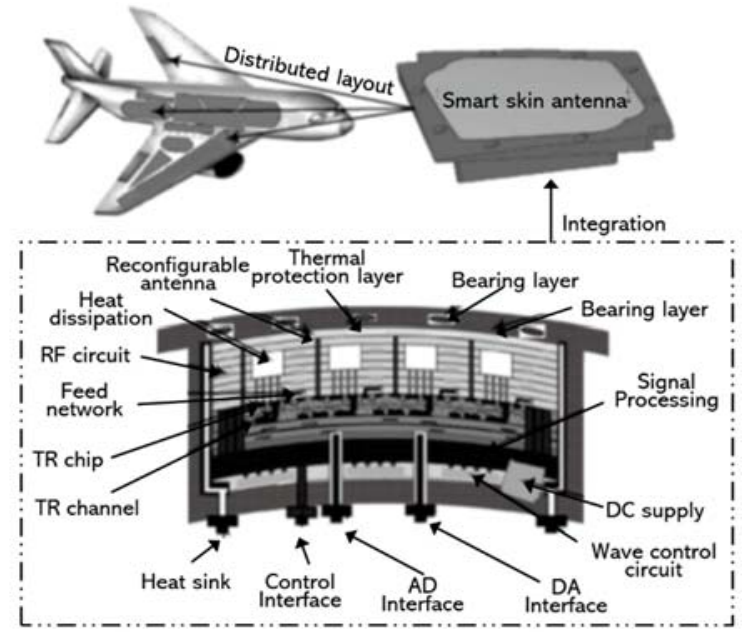

Figure 1. Typical smart skin antenna distributed layout.

3.1.2 Digital twin. Digital twin is a technical means to simulate, verify, predict and control the whole life cycle process of physical entities with the help of historical data, real-time data and algorithm model5. The application vision of digital twin technology for complex electronic equipment is shown in Figure 2. In the design stage of products, the accuracy of complex electronic equipment design can be improved and the cost of product $R \& D$ can be reduced by using digital twin. The performance and manufacturability of the complex electronic equipment can be verified by the design, simulation and Simulation of digital model. In the production stage, the production scheme verification, production process visualization and key index monitoring optimization based on the digital modelling of workshop can effectively improve the technologies such as 3D printing and emerging information technologies such as digital twin and knowledge engineering.

3.1.1 3D printing. At present, the application field of 3D printing technology is gradually extending from the medical, aviation and other industries to the electronic industry. According to relevant reports, the main research direction of the electronic industry is the manufacture of passive components such as surface printed circuits and inductors, and its application in complex electronic equipment is still under exploration 3 . With the rapid development of intelligent skin, the special manufacturing demand of smart skin antenna brings new opportunities for the application and development of 3D printing. The smart skin antenna shown in Figure 1 is a specific application of intelligent skin technology, and its most prominent feature is the integration of antenna and aircraft skin structure 4 . For the manufacturing of the conformal surface of the structure and function integration, the traditional technology has been difficult to realize. In the future, 3D printing will have a wide application space in the conformal manufacturing of intelligent skin and other new generation weapons and equipment.

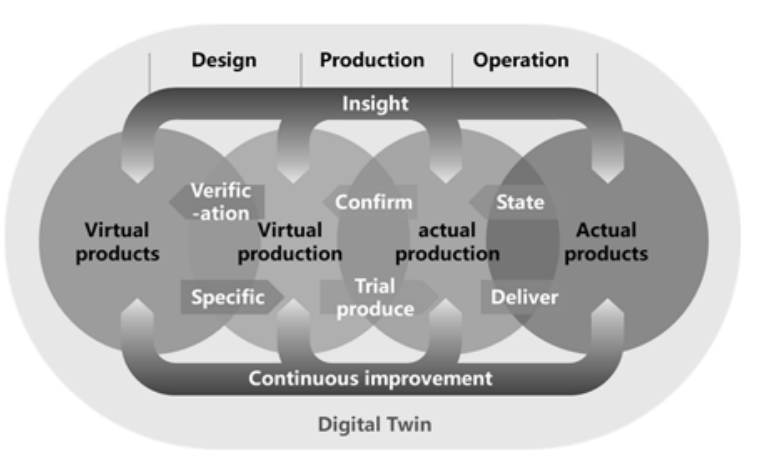

Figure 2. Digital twin vision of complex electronic equipment.

manufacturing quality and delivery speed of complex electronic equipment. In the stage of product operation and maintenance, by collecting the environment and status information of complex electronic equipment in the operation stage, remote monitoring and predictive maintenance are carried out to improve the user experience. At present, digital twin technology is still evolving rapidly, which will greatly promote the design, production and service of products.

3.1.3 Knowledge engineering. The core advantage of huge amount of industrial big data as important basis of intelligent manufacturing is production and application of "knowledge". For excavation of knowledge from knowledge big data, the data-to-information-toknowledge-to-wisdom (DIKW) Model is commonly used. 
Based on classic KIDW model, knowledge engineering is fused with management and equipment $R \& D$ process to form complicated electronic equipment R\&D DIKW model (Figure 3). First, R\&D data (D) are processed to form information (I), which is then refined to knowledge $(\mathrm{K})$, finally forming wisdom $(\mathrm{W})$, which is again applied

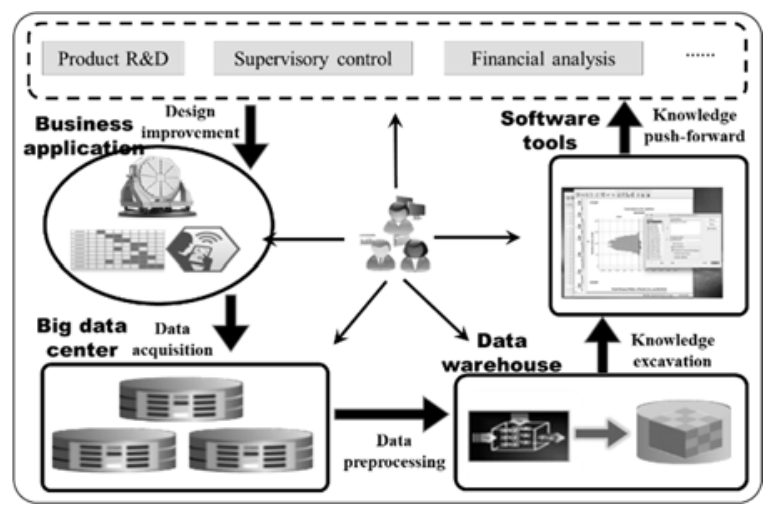

Figure 3. Complicated electronic equipment R\&D process DIKW model.

\subsection{Innovation of R\&D mode}

Virtual prototype is a computer-based simulation of a system or subsystem model with similar functions to physical prototype. The virtual prototype has the characteristics of low cost, fast speed, strong flexibility, high design quality and strong user experience. For complex electronic equipment, under the MBSE(ModelBased Systems Engineering) methodology, the construction of telecommunication virtual prototype and structure digital prototype is emphasized.

\subsubsection{Telecommunication virtual prototype.} Telecommunication virtual prototype of complex electronic equipment is a kind of mapping of physical prototype function and electrical performance in the computer, which can comprehensively and accurately reflect the characteristics and characteristics of real complex electronic equipment in terms of function and electrical performance. It can simulate and test in the virtual environment, replace the physical test to a certain extent, and optimize the telecommunication virtual prototype of complex electronic equipment according to the simulation results 6 . Radar telecommunication virtual prototype can be divided into concept prototype, function prototype and engineering prototype according to radar scientific research process and phased development results, as shown in Figure 4. Telecommunication virtual prototype can realize a new model centered R\&D mode, effectively improve the telecommunication performance in business to form new data (D); this creates a closed loop iterative work mode. In this way, through creation, accumulation, reuse, and sharing of knowledge, complicated electronic equipment scientific R\&D and production autonomous innovation capability is greatly improved.

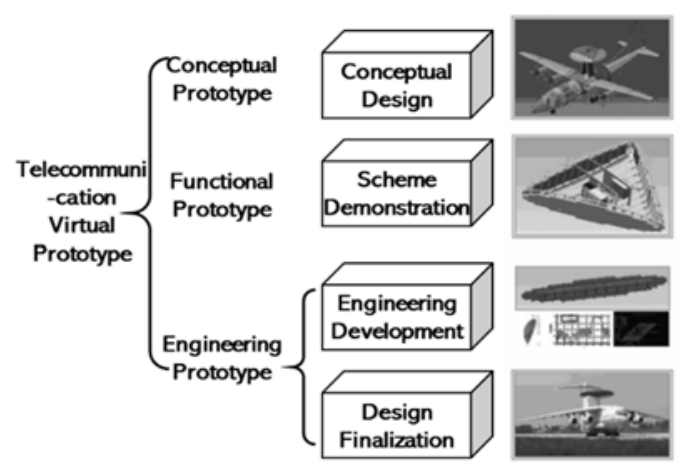

Figure 4. Telecom virtual prototype form of complex electronic equipment.

and quality of complex electronic equipment, shorten the development cycle and reduce the development cost.

3.2.2 Structure digital prototype. The application of structure digital prototype technology runs through the whole process of design, simulation and manufacturing of complex electronic equipment, including mechanical and thermal simulation analysis, 3D wiring, 3D labeling, interference inspection and maintainability inspection, virtual reality assembly and other processes, as shown in Figure 5. In the early design stage, according to the product demand analysis and electrical performance requirements, the system layout planning is carried out, the top-down design concept is adopted, and the collaborative design mode based on skeleton model is used to realize the rapid collaboration between the overall and subsystem. In the detailed design stage, according to the standard system of structural digital prototype, the design process of full three-dimensional modeling, full three-dimensional labeling, three-dimensional pipe routing, virtual assembly and interference checking of the digital prototype is implemented. At the same time, according to the digital prototype, the process side evaluates, reviews and designs the structural manufacturability of all levels of models in different depths, so that the design and process can cooperate in parallel. Finally, through virtual reality technology to get near real radar products, in the virtual system to achieve the new development of radar products operability, maintainability and other performance testing 1 . 


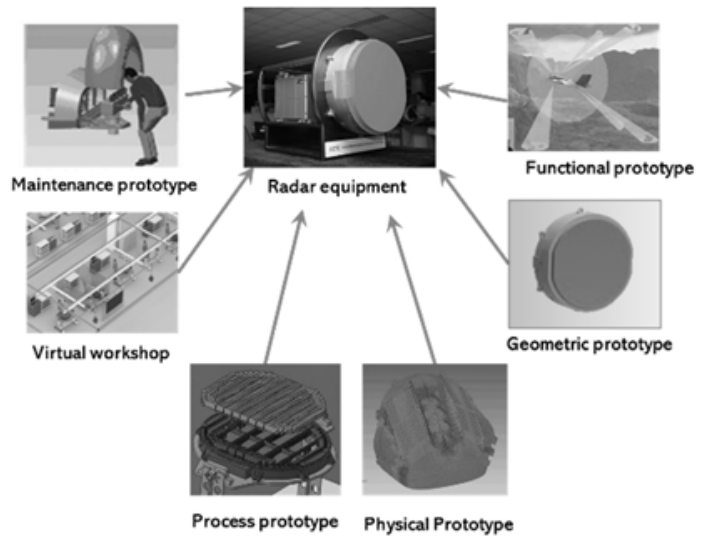

Figure 5. Digital prototype configuration of complex electronic equipment structure.

\subsection{Production organization innovation}

For the manufacture of complex electronic equipment such as radar, electronic countermeasure, communication and navigation, the industrial Internet technology can interconnect the production site, enterprise information system and collaborative platform, realize new production organization modes such as intelligent production within enterprises and supply chain collaboration among enterprises, and effectively stimulate the innovation vitality of manufacturing enterprises.

3.3.1 Digital Workshop. At present, for complex electronic equipment, the research of workshop integrated application technology based on digital twin is a hot field. The digital twin workshop of complex electronic equipment includes physical workshop, virtual workshop, workshop service system and twin data7, as shown in Figure 6. Before production execution, the virtual operation of digital twin workshop is used to effectively verify, analyze and optimize the production scheduling. In the process of production execution, the online simulation of virtual workshop driven by real-time data realizes the

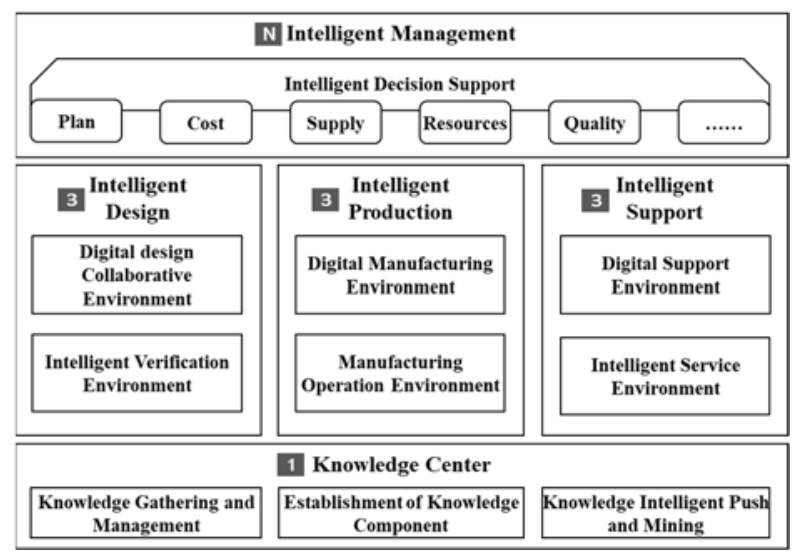

Figure 7. Complete solution architecture of Intelligent Enterprise Based on Model.

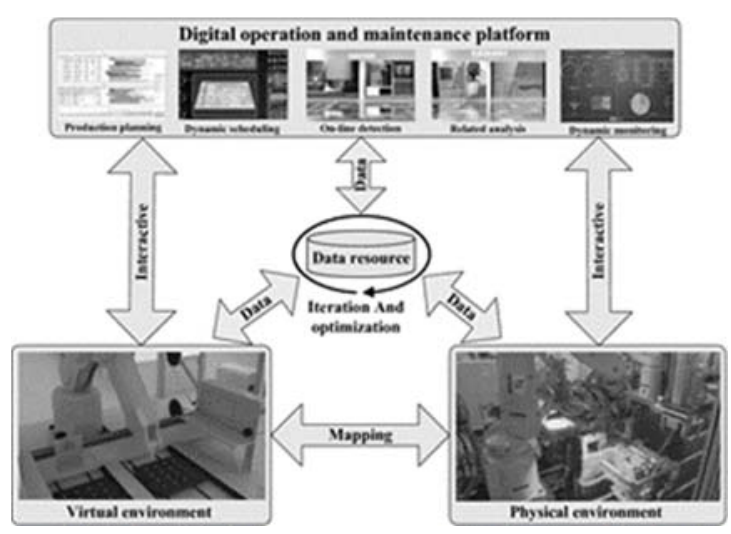

Figure 6. complex electronic equipment workshop architecture based on digital twin.

self-regulation of production process. After a period of production execution, data mining is carried out based on simulation data and monitoring data to support intelligent production decision and realize self-learning and selfoptimization of the model. Digital twin technology is effectively promoting the rapid improvement of complex electronic equipment design, production and support level.

3.3.2 Intelligent enterprise. Intelligent enterprise is a new management mode and organizational form after the digital transformation and intelligent application of enterprises. The typical complete solution of intelligent enterprise can be described by the " $3+\mathrm{N}+1$ " architecture as shown in Figure 7. "3" represents three end-to-end business processes in the product life cycle, corresponding to intelligent design, intelligent production and intelligent support solutions. " $\mathrm{N}$ " represents $\mathrm{n}$ top-down end-to-end enterprise management processes. "1" represents the establishment of a unified knowledge center for enterprises. The construction of model-based smart enterprise is a huge and complex system engineering, which involves enterprise organizational structure adjustment, business process optimization, information system application and so on.

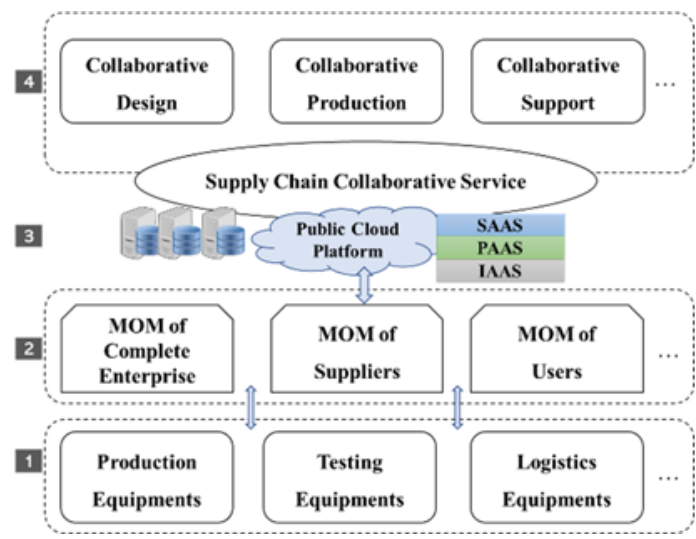

Figure 8. Architecture of supply chain collaboration platform. 
3.3.3 Collaborative supply chain. Complex electronic equipment has complex structure, long design cycle and many manufacturing links, which requires enterprises in the supply chain to carry out collaborative manufacturing. The basic architecture of the supply chain collaboration platform based on industrial Internet led by a complex electronic equipment enterprise is shown in Figure 8. The platform architecture includes field layer, control layer, platform layer and collaboration layer. Through supply chain collaboration, the value chain of complex electronic equipment manufacturing enterprises extends from a single manufacturing link to the upstream design and R\&D link, and to the downstream manufacturing control link, forming a network collaborative manufacturing system integrating engineering design, manufacturing, supply chain and enterprise management.

\section{Challenges facing complicated electronic equipment intelligent manufacturing}

At present, fast promotion of complicated electronic equipment intelligent manufacturing faces grim challenges in construction of standards system, fundamental R\&D capability, highly qualified composite type talents, network and information security, and construction of ecological system etc.

- Urgent need to establish industrial standards system. There are not many intelligent manufacturing standards for complex electronic equipment industry, and the advanced experience and successful cases in the industry need to be effectively promoted.

- Urgent need to improve fundamental R\&D capability. Core enabling products rely on imports, which hinders the independent development of intelligent manufacturing of complex electronic equipment to a certain extent.

- Urgent need to cultivate highly qualified composite type talents. The lack of industry leaders, interdisciplinary talents and compound skilled talents directly restricts the application and promotion of intelligent manufacturing system.

- Urgent need to solve network information security problem. Intelligent manufacturing changes manufacturing node from enclosed network environment toward open Internet environment, thus bringing new security challenge.

- Urgent need to improve intelligent manufacturing ecological system. No enterprise can possess all resources required for its operation and cooperation with other enterprises and enhanced industrial chain cooperation are necessary, to construct good industrial ecological system.

\section{Conclusions}

Information creates value and innovation drives the future. Intelligent manufacturing of complicated electronic equipment has formed new application mode innovation in full life cycle nodes of products such as application of technologies, R\&D mode, and production organization. It is exploration and practice of deep fusion between new generation information technology and advanced manufacturing technology. Effective operation and application of complicated electronic equipment intelligent manufacturing system will effectively shorten R\&D cycle, improve production efficiency and resources comprehensive utilization rate, and reduce operation costs and products disqualification rate. However, fast development of complicated electronic equipment intelligent manufacturing faces challenges in standards, talents, security, and ecology. The author believes that with constant progress of information technology and advanced manufacturing technology, as well as gradual implementation of state strong manufacturing strategy and strong network strategy, all current problems facing complicated electronic equipment manufacturing will be readily solved.

\section{Fund Projects}

National Defense Basic Scientific Research Program Project (JCKY2020210B006).

\section{References}

1. Changming Hu, Weizhong Cao, Changwu Wang, et al 2017 Exploration and Practice of Structure Digital Prototype of Complicated Electronic Equipment Electro-Mechanical Engineering 33(6) 1-9.

2. Kecun Ben, Zhanying Feng, Yaqi Cao Exploration and practice of intelligent manufacturing of electronic information equipment $[\mathrm{C}]$. Proceedings of Third International Workshop on Materials Science and Mechanical Engineering(IWMSME2020), 012056, 2020.

3. Zhou Dejian. Discussion and Analysis of Current Status of Electronic Products 3D Printing Technology Development $[\mathrm{J}]$. Electro-Mechanical Engineering, 2017, 33(2): 13-17.

4. LIU Xiuli, YUAN bo, SUN Fenglin. Application and Development of 3D Printing in Smart Skin Antenna[J]. Electronics Process Technology, 2020,41(06):311-313+332.

5. TAO Fei1,LIU Weiran1,LIU Jianhua. Digital twin and its potential application exploration[J]. Computer Integration Manufacturing Systems,2018,24(01):118.

6. Ming Li. 2017 A Study on Modeling and Simulation Technology of Radar Telecommunication Digital Prototype Modern Radar 39(04) 1-8

7. Changming $\mathrm{Hu}$, Wei Gao, Changhong Xu. Study on the Application of Digital Twin Technology in Complex Electronic Equipment [J]. Proceedings of the 7th Asia International Symposium on Mechatronics - Volume I, 2020: 123-137. 